\title{
Exploring Teenagers' Motivation to Exercise through Technology Probes
}

\author{
Helen M. Edwards \\ Sharon McDonald \\ Tingting Zhao \\ Department of Computing, Engineering and Technology, University of Sunderland \\ David Goldman Informatics Centre, St Peter's Campus, St Peter's Way, Sunderland, SR6 ODD, UK \\ \{helen.edwards, sharon.mcdonald, tingting.zhao\}@sunderland.ac.uk
}

\begin{abstract}
This study used existing digital technologies as probes to explore teenager's use of exerciserelated technology, their usage contexts, and their ideas for the design of ubiquitous technology that would persuade their peers to be more active in their daily lives. The teenagers formed two groups using two different types of step counters as their data collection device. Both groups used the same social website as their data logging tool and for social interaction. The one-week baseline plus six-weeks longitudinal study incorporated innovation workshops for generating usercentred design ideas: analysis of the findings drew out important lessons for the design of future devices. Key among these were: the impact of authority figures in restricting teenagers' use of technologies, teenagers' openness to sharing (privacy is not a concern), that data collection technologies must be ubiquitous but invisible, social interaction via technology is expected and positive messages reinforcing attainments of goals are valued (negative feedback is seen as demotivating).
\end{abstract}

Longitudinal study, teenagers, opportunistic exercise, technology probes, user-centred design.

\section{INTRODUCTION}

Recent advances in ubiquitous and persuasive digital technology offer new ways to encourage people to be more physically active (Ahtinen et al. 2010; Consolvo et al, 2006, 2008; Lin et al, 2006). Such interventions have sought to raise individual awareness of current activity levels by providing means to monitor and record daily activity. The issue of how to persuade has been addressed via the technologies themselves (presenting novel visualisations of the data) or through harnessing social influence within a group of users (Consolvo et al., 2006, Lin et al., 2006; Ahtinen et al., 2010). However, typically these studies reflect technological interventions that have been designed by adults, for adults. Research into persuasive digital technologies for children engaging in opportunistic (unplanned) everyday activity has been examined in few substantial studies. This is despite growing evidence of activity levels declining in adolescence, particularly among girls (Sirard \& Barr-Anderson, 2008). Therefore, younger people represent an interesting age group to work with for two reasons: technologies that appeal to adults may not appeal to them; moreover, their social environment differs considerably from that of adults (and that which adults experienced as teenagers themselves). It seems that teenagers rarely take a long-term view of how their current lifestyle may affect them in adulthood and as a result they may be less susceptible to persuasion regarding exercise. Moreover, the systematic review of De Meester et al. (2009) indicates that (non-technological) physical activity interventions with teenagers typically have had a short-term impact. Therefore, the challenge from a societal viewpoint is to find interventions with a long-term impact, which establish patterns of exercise behaviour in the teenage years that will stay with the individual in later life.

In line with this challenge, Sunderland City Council (as part of its ehealth theme within its national Digital Challenge project) wished to assess whether providing exercise-focused digital technologies to typical adolescents within the city would have an impact on their behaviour and attitude to physical activity. This paper reports on the study the authors were commissioned to do on their behalf. The focus was on generally healthy children since maintaining their activity levels through adolescence and on into adulthood was seen as providing the greatest societal health benefit: it is known that in this group activity levels decline substantially resulting in inactive adults. We adopted Hutchinson et al's (2003) technology probes approach to gain an understanding of the users and their contexts, to field test the technology and to elicit new design ideas from the users. 


\section{TECHNOLOGY TO PROMOTE ACTIVITY.}

It seems to be generally accepted that digital technologies that are used to promote behavioural change need to raise individual awareness of current activity levels. Our review of the literature has shown that this is typically achieved through standard, or mobile phone-based, pedometers or accelerometers e.g.(Consolvo et al, 2006; Ahtinen et al., 2010; Lin et al., 2006). Interesting research questions then emerge in terms of identifying the most persuasive mechanisms to feedback this data to users in the hope that they will either maintain existing activity levels or increase them further. Researchers have investigated the use of fitness journal applications such as Consolvo et al's (2006) Houston system, and more novel visual representations of activity data. Other approaches include representing activity as progress on a virtual trip (Ahtinen et al., 2010); linking activity to the growth of virtual pets (Lin et al's 2006 Fish N Steps) and environments (Consolvo et al's 2008 UbiFit Garden). Persuasion may also be achieved through the use of social influence by the act of sharing an individual's data among a group of users (Consolvo et al., 2006; Lin et al., 2006; Ahtinen et al., 2010). Depending upon the people involved, this could create an environment of mutual support and/or healthy competition.

Deriving a clear picture of the success of these interventions is difficult, not simply because of differences in the technologies used, but because of differences in the baseline attitudes and activity levels of participants, and the length of the evaluative trials. The common theme drawing these studies together is that they focus on technology that has been designed by adults for adults. Children and teenagers may draw motivation from different sources; therefore it is unclear as to what type of approach may work with younger people.

Some tentative suggestions were made by Toscos et al. (2008) that technology may help teenage girls become more physically active where it harnesses the power of existing friendship groups. Their mobile application "Chick Clique" allows up to four friends to track and share their walking activity, and send supportive and encouraging texts to one another. Over a field trial of two weeks the average step counts of the eight participants increased, suggesting that data sharing engendered a sense of healthy competition. However, the girls (all aged 13) had difficulty in constructing supportive messages. Perhaps suggesting that for effective social support a level of maturity is required.

The studies cited above have, in the main, adopted user-centred design practices. Designs have been created on the basis of data gathered about users from interviews and questionnaires and prototype systems have been developed and evaluated. Some researchers have taken further design inspiration from the psychological literature (Consolvo et al., 2009) others from the sociomedical literature (Toscos et al., 2008).

For this, our first foray into the field of exercise related technologies, we wanted to take a slightly different approach and use "technology probes" (Hutchinson et al., 2003) as an approach to usercentred design. According to Hutchinson et al. (2003) "they are not a prototype but a tool to help determine which kinds of technologies would be interesting to design in the future" (Hutchinson et al. $2003 p$ 18). They therefore suited our purpose in that the three goals of technology probes: to understand users and contexts, to field test technology and to inspire design ideas, mapped our purpose of wanting to discover the type of exercise-related technologies that would appeal to teenagers; the nature of their contexts, the challenges they face in the use of such technology and their ideas for the design of exercise-related technology. In the sections that follow we describe, the design of our study, the technology probes used and discuss our findings.

\section{STUDY DESIGN}

\subsection{Participants}

The participants required for this study needed to be generally fit and healthy adolescents who could provide user-centred insights into the digital technologies that they would see as persuasive in helping them establish and maintain an active lifestyle, for long-term health benefits. We contacted more than 50 voluntary youth organisations in the city and provided information about the project (including the incentive of a gift worth $£ 100$ as reward for those completing the project). A small number of groups responded positively and with their help we recruited the participants for the project. In this way we gained both the study participants and also access to specific youth workers with established roles as authority figures in the participants' lives which eased aspects of running the project.

We recruited 18 participants, 12 of whom formed two teenaged groups where social links would be important, the remaining six participants were younger in age (11-13) and operated in an individual manner (we do not report on the data from this third group in this paper). We obtained university ethical approval, for this study and, since we were working with adolescents, acquired CRB (Criminal Records Bureau) clearance. We also ensured we gained explicit participant and parental/ carer consent. 
The two teenaged groups were: Group A (three girls and three boys, all were 14 years old) and Group B (two girls and four boys, five were 13 years old and one boy was 15). Group A members were all friends and attended the same school and youth club; Group B members had looser ties, but all were already friendly with at least two group members.

\subsection{Technology probes}

Two different types of technology probes were used: one for step data collection and one for data logging.

\subsubsection{The data collection probes}

The data collection probes were Nintendo's "Walk with Me! "TM" activity meters for Group A which linked to Nintendo DS-Lite ${ }^{\mathrm{TM}}$ consoles and the "Walk with Me! TM" game (since the city council specifically wanted an evaluation of whether such devices could have a positive impact on activity) ${ }^{1}$. "Walk with Me! ${ }^{\mathrm{TM}}$ " was chosen since, unlike other reviewed games, it did not emphasise weight loss (we saw this as important in working with the adolescents given the prevalent media focus on unrealistic body images). The activity meters came as a pair, so each member of Group A chose a "friend" to use the other meter. The data collection probes for Group B were Omron Walking Style II pedometers (with acceleration sensor technology) The authors had investigated a number of pedometers and found these to have acceptable accuracy for this project (since it was not dependent on highly accurate exercise data).

\subsubsection{The data logging probe}

From the analysis of the literature we had identified a number of design features that needed to be built into a data logging probe. These were:

- The social dimension: support and competition.

- The facility to record daily: (i) step counts, (ii) activities not captured by the data collection probe and (iii) barriers to activity.

- The option to keep data private or shared.

To provide a technology probe that supported these options we constructed a social networking website (ehealth-elgg) using the elgg ("facebooklike") environment (http://elgg.org) with two closed communities to ensure child-protection. Elgg supports personal presence via members' profiles and blogs, plus social interaction via personal messages, wall posts and wire posts (short messages to the entire group). To encourage competition we customised the site to send daily and weekly rewards to those achieving their targets and publicised these to the wire (this satisfied our

\footnotetext{
${ }^{1}$ N.B. Walk with Me! TM has features that are not reported here
}

first requirement). We added functionality to enable daily records to be logged by the participants (satisfying our second requirement) and allowing them to keep these data private or public (satisfying our third requirement). Figure 1 shows an anonymised user profile for ehealth-elgg.

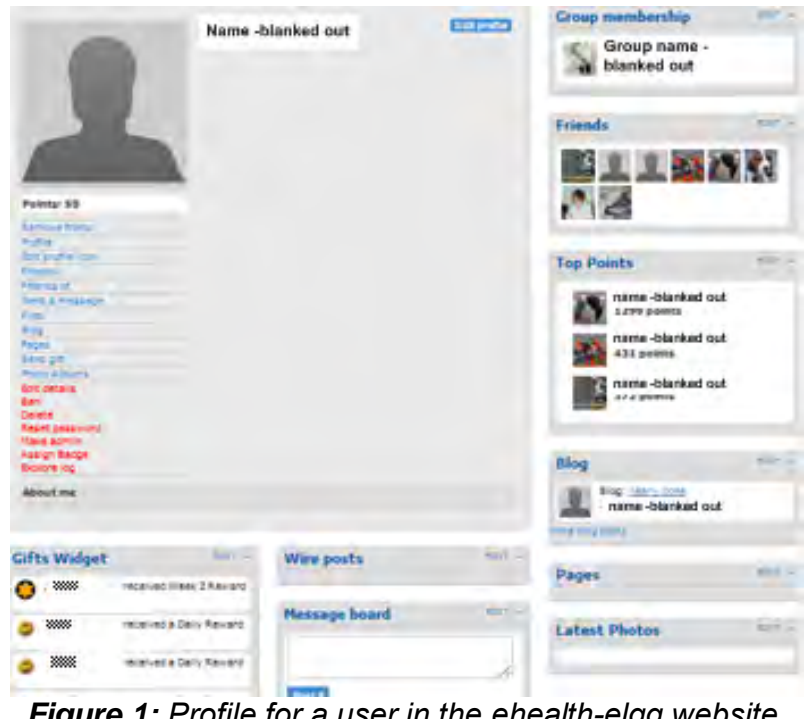

Figure 1: Profile for a user in the ehealth-elgg website

\subsection{Process}

The study had a baseline week plus the six weeks main study (including midpoint innovation workshops) and a final assessment session.

\subsubsection{Baseline week}

At the beginning of the baseline week, we held meetings with each group to introduce the study and we asked them to complete a set of questionnaires. These collected information on their attitudes to physical exercise (and the link with self-image), their technological experience and their views on their current activity levels: these are discussed in Section 4.1. A parents/carers' questionnaire was also used to gain insight into the family attitudes to exercise. Finally, a pedometer was provided (and calibrated for each participant) and an explanation given of how it should be used during the subsequent week. The participants used the pedometer throughout each day and recorded their daily steps count plus any additional activity not captured by the device. This data was used to determine whether the standard 10,000 daily steps target was reasonable for the main study. It also provided a baseline from which to track any changes in activity levels as a result of the intervention.

\subsubsection{Main study phase}

We met with the groups at the start of the main study to collect the pedometers and recorded baseline data, introduce the technology probes and clarify the study's purpose. We emphasised that the aim of the study was to get their reactions to 
the technologies and to help us understand what technologies would be attractive to typical users of their age group. We alerted them to the planned innovation workshop where we would seek their design ideas. The introduction of any technology changes the context, so to minimise the effect we had on the study, we made it clear that we had nothing invested in the technologies provided and were just using them as probes. This approach differs from the reviewed studies where technologies had been designed, and were being evaluated, for their effectiveness in changing exercise behaviour. Over the following six weeks, the participants captured and recorded their daily step count and other physical activity. We also tracked their level usage of the website and the purposes for which they used it.

\subsubsection{Innovation workshop}

The design innovation workshop held with each group explored the participants' experiences and reactions to the study, and elicited design ideas for new technologies that might support (and encourage) opportunistic (unplanned) exercise. We developed posters as additional probes representing potential software and hardware artefacts (as shown in Figure 2). These suggested applications that would access step data for a user's benefit within a game. In particular we provided visuals of (i) social games where activity rewards could be used personally or collaboratively, and (ii) applications with a different locus of control where the benefit would enhance the product. A third visual suggested linking activity to existing video games thus binding physical activity into a game of choice for enhanced gameplay. The hardware poster focused on what data might be captured, what the devices might look like, and how they might be worn.

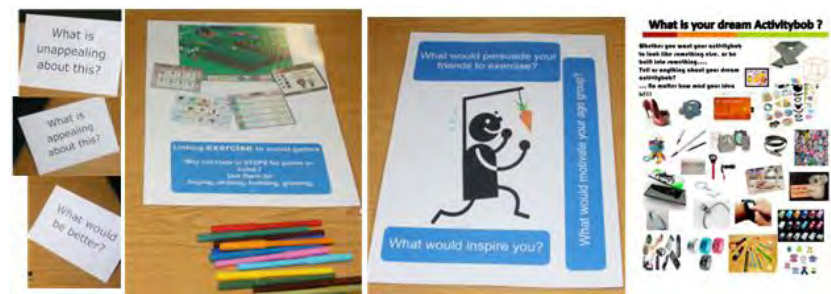

Figure 2: Sample posters and probes used in innovation workshops

The benefit of providing visual stimulants is a rapid generation of ideas for debate, then acceptance or rejection; but the disadvantage is that this can blinker participants into thinking about what has been presented and not what "could be". To account for these issues we operated the two innovation workshops in different orders as shown in Figures 3 and 4 . In both workshops the participants were encouraged to first capture their ideas pictorially or textually and then engage in group discussions to explore ideas further.

\subsubsection{Final assessment}

At the end of the study interviews were held with participants and the baseline questionnaires were re-administered in order to identify any changes. Finally the teenagers were given their rewards (a gift of $£ 100$ value) for participating throughout the full study and thanked for their participation.

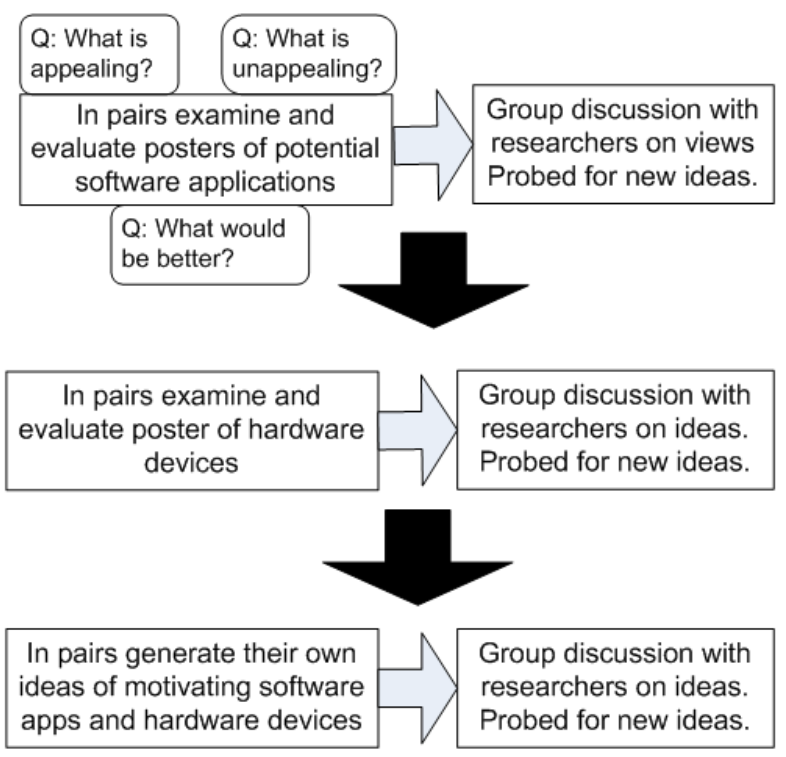

Figure 3: Group A's innovation workshop process

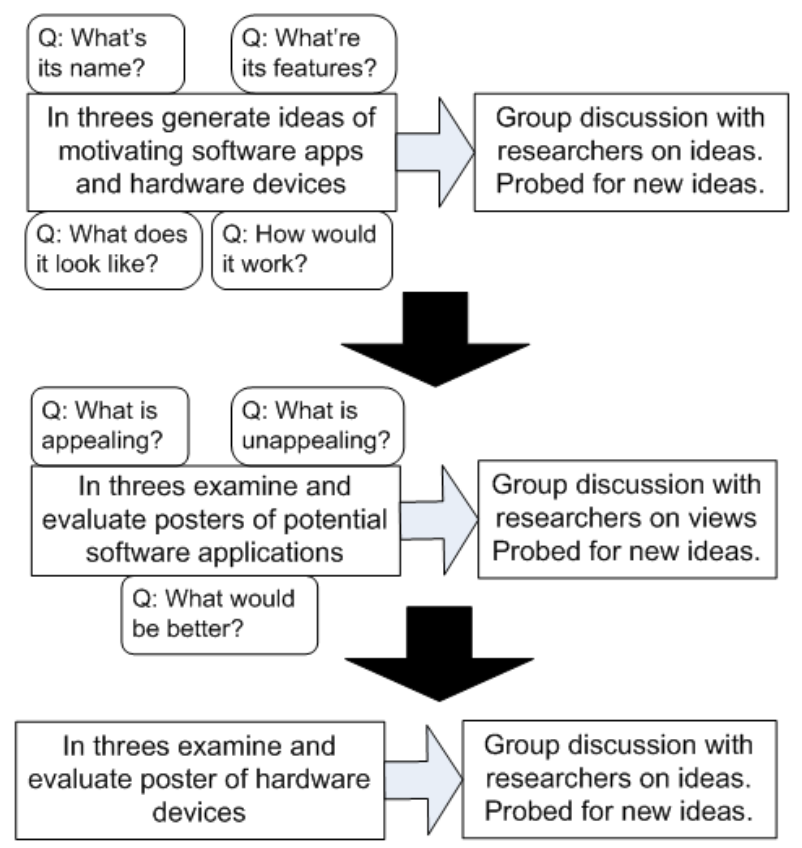

Figure 4: Group B's innovation workshop process.

\section{RESULTS}

Numerous sets of data were collected: these encompassed attitudinal and factual data, qualitative and quantitative data. Questionnaires, interviews, focus groups, probes and digital 
technologies were used to collect the data. In this section we present our findings.

\subsection{Perceived activity and attitude (baseline and final assessment comparisons from questionnaire data)}

\subsubsection{Technology usage}

All 12 participants were experienced computer users with at least five years experience, and all used them several times per week or daily. The main use was social (typically internet-browsing) with less usage for homework purposes. 11 owned games consoles, which were used less frequently: usage was split between individual and social play with family and friends. Sports and music games were the most popular genres. All participants owned mobile phones and half were aware of health and exercise applications for these.

\subsubsection{Attitude to exercise}

The attitude to exercise questionnaire was based on existing instruments by Whitehead (1995), Tobe (2005) and Sarkin et al. (2001). Quantitative factual data were collected plus attitudinal data using Likert scale questions (for these the directions of statements were varied to ensure we did not lead the participants in their responses). Using SPSS and a range of non-parametric statistical techniques, we analysed the quantitative data however, there were few results of statistical significance. The items analysed included: motivation to and enjoyment of exercise, perception of sport, physical self-perception, and exercise environment.

However, the data collected indicated that, all participants had positive views on the importance of exercise for health and impact on appearance (providing scores that indicated agreement or strong agreement), and all believed they needed to do exercise. Two were neutral about whether there was a link to a good appearance and two were neutral to the idea of exercise being fun: but all other respondents agreed or strongly agreed with the positive aspects of exercise. In terms of organised sport only two expressed a preference for watching rather than taking part (five were neutral) and confidence levels were high: half believed they did well at any new sport, and assessed themselves as good at sports in general. However, only two expressed a preference for exercise over watching TV.

The Transtheoretical Model, TTM, (Sarkin et al., 2001, Lin et al. 2006) was used to assess commitment to exercise: 11 ranked themselves highly (in the action to termination stages), the twelfth positioned himself in the preparation stage (the third of six stages). Non-parametric analysis revealed no differences in the TTM ratings for the groups $(Z=-1.134, p=0.257)$, and no individual differences across the groups were detected.

This age group (13-15) is often considered to be negatively concerned with self-image; therefore we explored their attitudes to confidence and appearance: the results are presented in Figure 5 showing generally self-confidence and selfperception within the groups.

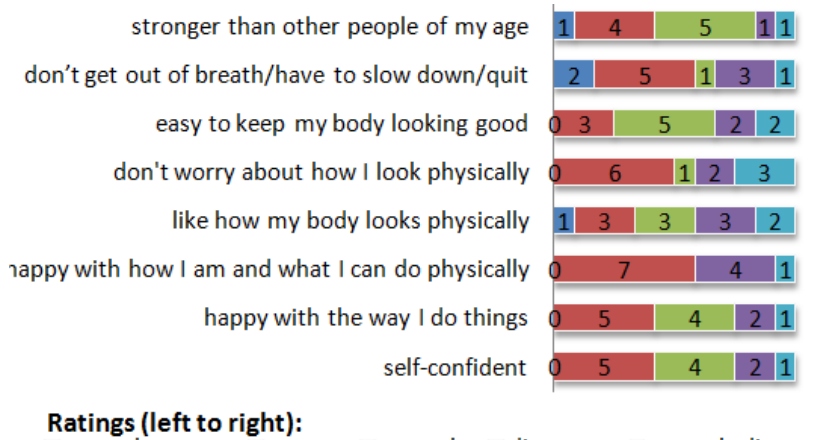

w strongly agree agree neutral wisagree w strongly disagree

Figure 5: Participants' profile for self-perception (rated from strongly agree to strongly disagree)

For most questions there was a spread of responses, but few issues that had a predominantly negative response set. The high-level questions about confidence and ability to do things only had three participants disagreeing; the most negative views five (strongly) disagreeing about liking how they looked. We explored the extent to which participants had the motivation and opportunity to maintaining a healthy level of exercise, the data is presented in Figure 6. Overall personal motivation was not seen as an issue, although most liked to exercise with friends (although only one thought that all of their friends liked exercising) they did not perceive (structured) exercise as a good way for meeting new people. The opportunity to exercise existed (in terms of time and energy) but perhaps the motivation was lacking since only three tried to make time for exercise. The response sets show the participants to be generally active with positive attitudes regarding exercise and its importance.

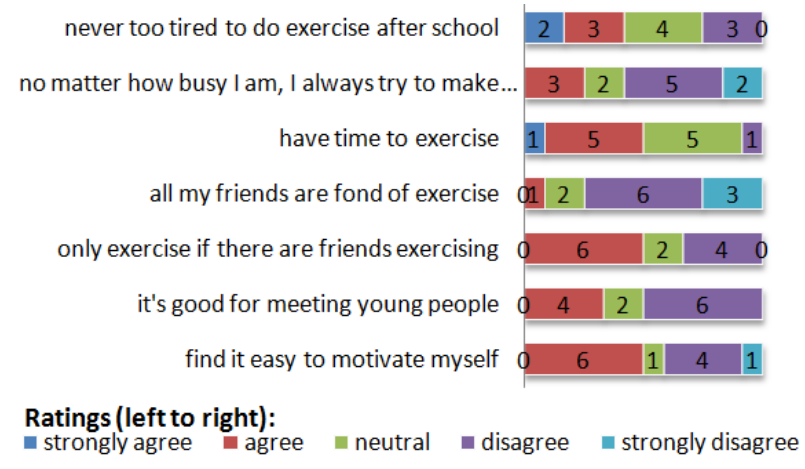

Figure 6: Participants' motivation to exercise, (rated from strongly agree to strongly disagree) 
We reissued this questionnaire at the end of the study, the data were statistically analysed and no significant changes were identified.

\subsubsection{Physical activity profile}

A variant of Kowalski et al's (1997) Physical Activity Questionnaire, PAQ, (developed for children aged 8-14) was used. PAQ reviews the previous week's activities to form a snapshot of typical physical activity. In the version used we replaced non-UK sports with those that are more commonly encountered (for instance removing baseball and inserting rugby and netball), we also provided the opportunity for missing sports to be added by the participants. The results in Table 1 show that walking, jogging and football (soccer) were the most popular activities and were undertaken most frequently. These are all activities that require little equipment and can be adopted opportunistically.

Table 1: Participants' baseline activities over one week.

\begin{tabular}{|l|c|c|c|c|}
\hline & $\begin{array}{c}\mathbf{1 - 2} \\
\text { times }\end{array}$ & $\begin{array}{c}\mathbf{3 - 4} \\
\text { times }\end{array}$ & $\begin{array}{c}\mathbf{5 - 6} \\
\text { times }\end{array}$ & $\begin{array}{c}\mathbf{> 6} \\
\text { times }\end{array}$ \\
\hline Walking & 1 & 1 & & 6 \\
\hline Jogging or Running & 4 & 2 & 2 & \\
\hline Football (soccer) & 3 & 1 & & 3 \\
\hline Skipping & 2 & 1 & & \\
\hline Bicycling & 2 & & & \\
\hline Aerobics & 2 & & & \\
\hline Swimming & 2 & & & \\
\hline Dance & 1 & & 2 & \\
\hline Basketball & 2 & 1 & & \\
\hline
\end{tabular}

Since the participants all attended school it was useful to know how they used their opportunities for exercise within the school day. Table 2 shows that short breaks were rarely exploited and even the longer lunchtime break was a time of little activity

Table 2 Activity levels for participants during school day

\begin{tabular}{|l|c|c|c|}
\hline & Lunchtime & Break & P.E class \\
\hline Very active & 2 & & 4 \\
\hline Active & 1 & & 7 \\
\hline Some activity & 1 & 3 & \\
\hline Little activity & 7 & 9 & \\
\hline Inactive & 1 & & 1 \\
\hline
\end{tabular}

In their free time, the teenagers had more control over their activities, Sunday was seen by them as their "day of rest" with least strenuous activity and, as shown in Table 3, their highest level of activity occurred immediately after school.

Table 3: Number of times involved in activity outside school by participants.

\begin{tabular}{|l|l|l|l|l|l|}
\hline \multicolumn{1}{|c|}{ Activity count } & $\mathbf{1}$ & $\mathbf{2}$ & $\mathbf{3}$ & $\mathbf{4}$ & $\mathbf{> 4}$ \\
\hline Straight after school & 6 & & 3 & 2 & 1 \\
\hline In evenings & 3 & 3 & 3 & 3 & \\
\hline During the weekend & 4 & 5 & 3 & & \\
\hline
\end{tabular}

At the end of the study this questionnaire was reissued: again, non-parametric statistical analyses showed no significant changes.

\subsubsection{Parents' assessment}

The parents/carers' questionnaire triangulated the adolescents' view of exercise and also identified the support available to them. In general their responses were in line with the teenagers'. They reported the main barriers to exercise as being too much homework (8), no one to exercise with (7) and that friends didn't like exercise (1).

\subsection{Step counts, activity and barriers during study (participants' recorded data)}

\subsubsection{Step counts and activity}

The steps data collected over the longitudinal study (as summarised in Figure 7) suggest a slight downward trend for both groups and this pattern is reproduced within individuals' data.

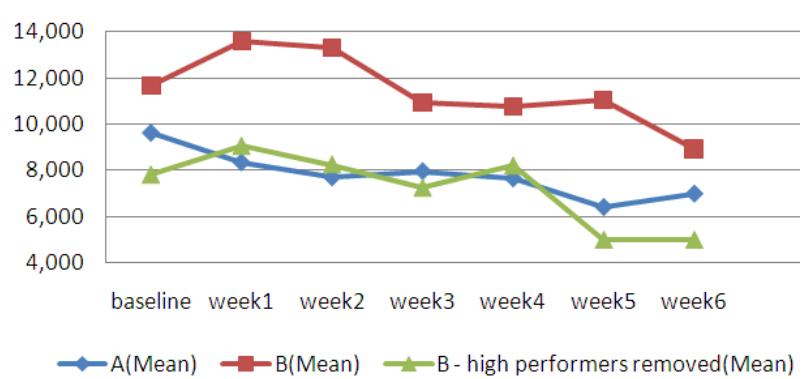

Figure 7: Mean daily step count by week for groups.

During the study there was one week's school holiday, which we believed might affect step rates. However, our data analysis showed no significant differences during the holiday period. Group B's study period also coincided with two weeks of unusually severe snowfalls, but again, there was no consistent pattern of change in step rates. Looking at the steps data in more detail (in Table 4) we can see variations in the extent to which the participants achieved their daily targets. Seven reached their target achieved more (in percentage terms) during the baseline week than over the main study. Perhaps the motivation to maintain the steps level over six weeks was a much harder challenge.

Table 4: Percentage of time steps targets reached.

\begin{tabular}{|c|c|c|c|c|c|c|}
\hline Participant & Ag1 & Ag2 & Ag3 & Ab1 & $\mathbf{A b 2}$ & Ab3 \\
\hline Baseline & 14 & 29 & 43 & 57 & 71 & 29 \\
\hline Main study & 14 & 10 & 5 & 52 & 50 & \\
\hline Weekend-d & 20 & 13 & & $\begin{array}{l}60 \\
29\end{array}$ & $\begin{array}{l}63 \\
-14\end{array}$ & 14 \\
\hline Participant & Bg1 & Bg2 & Bb1 & Bb2 & Bb3 & Bb4 \\
\hline Baseline & 43 & 14 & 29 & 71 & 100 & 29 \\
\hline $\begin{array}{l}\text { Main stuo } \\
\text { Weékday } \\
\text { We-ékend }\end{array}$ & 21 & & $\begin{array}{r}-\frac{33}{33} \\
-\frac{23}{29}\end{array}$ & $\frac{88}{6} \frac{3}{64}$ & $\frac{90}{100}$ & $-\frac{40}{50}$ \\
\hline
\end{tabular}


10 of the 12 achieved their targets more successfully over weekdays than during weekends: indicating perhaps that their school life keeps them active. This is in line with their self-assessed activity levels as shown in Table 3. Qualitative feedback from interviews indicated that some liked to have "lazy days", but in contrast one teenager considered the weekend to be the only period when she could devote time to an activity of her choice (dance sessions of up to five hours duration). However, the design of the activity meter used meant that it was impractical to wear during dance and therefore the steps from this high-level exercise were lost.

\subsubsection{Barriers}

Using the website participants logged daily barriers to doing and monitoring exercise. The statements were transcribed and an affinity diagram was built to identify the main themes of:

- Problems with recording full number of daily steps accurately: these occurred because of forgetting to wear the device (5), ineffective data recording by the device (4), inability to wear during an activity (4), loss of the device (2) prevented from wearing by someone in authority (1).

- Barriers to activity: these occurred because of snow and rain (5), illness (4), homework (3) and holidays (3).

- Choosing not to be active: two participants reported having a "lazy day" on occasion.

\subsection{Final assessments}

\subsubsection{Step activity}

Four of Group A and two of Group B said they would continue to monitor their steps after the project end, two of both Groups A and B were undecided and two of Group B had decided not to continue. Interestingly half the participants (in both groups) believed that their physical activity had increased as a result of the monitoring and only two, in Group B, were sure that it had not. This contrasts with the factual steps data (discussed in section 4.2 ), which saw a gentle decline in activity.

\subsubsection{Social support and competition}

Different views were expressed about willingness to share data. One Group A member preferred not to share his since he felt ashamed that he had not taken as many steps as the others. The other five group members were open to sharing their data, but expressed similar reservations about sharing low step counts "...depends how many steps I did that day". Group B members seemed much less concerned about others seeing their steps "I don't mind who sees the option", "I'm proud of the steps I did, I want others to see". Group A members were more interested than Group B members in seeing their group members' data. A Mann-Whitney test revealed that this difference was significant $(Z=-$
2.068, p<0.04) Group A (mean 3) were more interested in seeing each other's data than Group B (mean 2.17). This difference in social interaction was also evident in comparing the two groups' activity within the website. The number of communications with the group or individuals for Group A was (for each member) 61, 54, 34, 31, 20 and 1 . Whereas the comparable numbers of communications for Group B were 16, 6, 1, 0, 0, 0. This sharing with, and interest in, one another may reflect the stronger friendship ties between Group $A$ than Group B.

\subsubsection{Rewards and motivation}

Participants using the website were asked to indicate if it had created a sense of competition, a Mann-Whitney test revealed $(Z=-2.142, p<0.032)$ that Group A indicated that the site created competition significantly more (mean rating 3.83 ) than Group B (mean rating 2). The competition did not appear to stem from the rewards issued for achieving targets as there were no differences between the two groups in terms of the impact of rewards on motivation to be active. Again, it may be that the existing social bond between members of Group A was the overriding factor here.

\subsection{Feedback on technology probes}

In the innovation workshops we not only gathered design ideas but also feedback on the technology probes used in the study. The data sets were analysed using card sorts and affinity diagrams.

\subsubsection{Feedback on activity meters}

The emergent themes for the activity meter were:

Wearability: flexibility was needed in positioning the device around the body

Lack of accuracy: in recording step counts (one girl reported "the activity meter is only picking up steps from one leg!')

Data visibility: for those using activity meters step counts could only be seen once data had been uploaded to the console.

Integration with other technologies: such as the wii.

\subsubsection{Requirements of activity devices}

Our participants wanted activity devices to record data from a range of activities (such as bicycling, badminton, swimming). Although it can be argued that the devices used could record during some of these activities (such as running, tennis), problems were experienced with wearing and keeping devices in place during strenuous activities "...I tried keeping it in my goalie gloves". Seven participants had typically kept the device in a pocket, four on a clip and one in a bag, but this was not always suitable. Seven wanted redesign for wear-ability, six wanted more discreet devices.

Not all exercise can be measured via steps therefore seven participants suggested heart rates 
could be measured and the five girls plus one boy wanted to know the calorific value of their activities. Other functionality that was of interest included calculation of the average walking speed (six participants), calculation of distance covered (all of Group A and the two girls in Group B) and a record of routes walked (five participants).

\subsection{Design ideas for future devices and applications}

The participants' evaluation of the research team's ideas generated the following themes.

\subsubsection{Linking steps to standard digital games}

Using step counts as rewards for existing digital games was generally an appealing concept, which generated the following themes:

Game content and genres: for this to appeal widely various genres would need to be included from action-based to collaborative games. "...girls don't really like war and action games so you've got to make them unisex"

"It's cheating": the positive view of this was that advantages in a game would be won through physical activity, but some worried that a it might undermine the point of playing the game.

\subsubsection{Linking steps to virtual pet game:}

Here the emergent themes were:

Competition: "guilt-tripping" participants into exercising after comparing their pet to others'.

Target audience: this would only attract a subset of people, "... girls, under eights".

Boredom: variety is needed otherwise users would abandon the game.

Value of the pet: there is emotional buy-in which may have a positive impact (in that the pet benefits from users' activity); but there are demotivating costs "...if I'm too busy my dog might die".

\subsubsection{Link steps to social games}

This idea was generally highly rated, although one boy commented "... I'm not bothered what my friends are doing as long as l'm doing well'. The emergent themes were:

Social Collaboration: communication needs to built in, friends can work together "... I like to be able to share with friends".

Appropriate content: variety is needed, suggestions include farms, restaurants, building hotels, building an army.

Negative consequences (such as crops withering) need to be avoided as they demotivate.

Rewards are desirable: to convert activity into points for "buying things".

Platform accessibility: it is important that any game is accessible so it was suggested that is it would be better on a gaming console than being reliant on access to the Internet.

\subsubsection{Participants' own design ideas for future devices and applications}

All of the features that emerged from reviewing their experiences and evaluating the research teams' probes re-emerged when the groups were probed for their novel ideas. However, there were some additional emergent concepts. These were:

Integration into existing objects: particularly items already used - e.g. mobile phone, keys.

Use of "negative" persuasion: sufficient exercise is needed to unlock something that is wanted "... play your ipod music but only when you do activity",

Use of "positive" persuasion: rewards link to energy levels "...a text sent to your phone saying a sport and a length of time, if the person does it, there will be phone credit topped up on the phone"

Use of "kinetic" persuasion: exercise powers some physical appliance "... the more steps, the more battery power you have for your phone"

Individualistic: egocentric approaches focusing on recording the individual and broadcasting to others Real world rewards: providing discounts, "freebies", financial rewards.

\section{DISCUSSION}

The technology probes enabled us to learn about teenagers, technology and exercise in terms of users in context, field testing the technologies and inspiring users to think about new technologies. Therefore we present our discussion under these headings, drawing on the results from study and innovation workshops.

\subsection{Users in context}

The data collection devices provoke different reactions in different people. For instance, on receiving the pedometers the boys in Group A immediately started running and jumping around the room to test their accuracy and compete with one another in their steps total. Whereas the girls sat in a social group investigating how to use the device. In everyday use the devices needed to be able to adapt to the context in which they were being used; and this context can change within a day (for instance from walking to cycling). Participants in the reviewed studies (for instance Consolvo et al. (2006), Lin et al. (2006), Toscos et al. (2008)) identified the same themes of wearability, visibility, inaccuracy and inability to record non-step based activity.

The social context within which the teenagers operate is of interest. As individuals the teenagers in this study did not have significant concerns about their self-image and were generally self-confident; moreover, they seem to belong to a generation that considers sharing the minutiae of their daily lives via various media as the norm and this sharing extends beyond close circles of friends to a much 
wider public: thus privacy is not a concern. Most participants liked seeing the group members' data displayed, although they were not interested in the rankings. Social competition was not important unlike Toscos et al's (2008) teenagers (existing friends) who saw sharing of achievements as providing healthy competition and motivation in the short term. In Arteaga et al.'s (2010) study the teenagers shared achievements with their choice of people, but no particular effect was identified. Toscos et al. (2008) also identified a positive effect of social support amongst their participants (provided via text messages), however this was affected by the quality of the messages sent. For adult study groups social support has been assessed as having an overall positive impact in those studies where social bonds already existed (Ahtinen et al., 2010; Consolvo et al., 2006). In contrast Lin et al's (2006) participants, who were not friends rarely used the anonymised social chat facility. However, in each of the adult studies reviewed, social competition has been assessed as having an overall positive impact (Ahtinen et al., 2010; Consolvo et al., 2006; Lin et al., 2006).

The family played an important role in our study. Support from family members (or a significant adult in their lives) was important in maintaining the participants' involvement in our study. Moreover, the engagement of family members as "Walk with me! "TM" friends acted as a catalyst in some families increasing their overall exercise levels. Some of those who chose a family member reported subsequently a beneficial impact on the family's activity levels.

Factors outside these social groups can affect exercise levels and the freedom to monitor performance. For our groups the factor that most significantly inhibited exercise was the school workload accompanying their two-years GCSE study and which began to build in the pre-GCSE year (around age 13). The second key factor was different authority figures (such as teachers) who had control over what devices they could use, where and when.

\subsection{Field testing the technology}

Feedback suggests that users will only be motivated to collect and log data when they are confident that the devices they use are accurate and capable of capturing more than just steps data. But the requirement to view this data varies: some Group B members were pleased that the activity meter did not display their steps count, whereas others were frustrated by this same feature, indicating the need for customisation. The wearability of the data capture devices is also a key concern and the mechanism for effectively uploading and storing the captured data needs to be seamless and automatic (for both user satisfaction and research purposes).

During the study there was a one-week school holiday, which could have affected step rates. However, data analysis showed no significant differences during the holiday period. Group B's study period also coincided with two weeks of severe snowfalls, but again, there was no consistent pattern of change in step rates. This seems to be in contradiction to Consolvo et al.'s (2008) study that commented on the impact of the season in their study (which included Thanksgiving, Christmas, New Year, poor weather and dark nights). Their participants' stated that the UbiFit Garden had motivated them to overcome seasonal inertia.

Working with users of this age group is very different from working with adults, either in the field or lab. We were not perceived as authority figures so the substantial support provided by the youth leaders and parents/carers was key in keeping the teenagers engaged. Another unexpected obstacle in the smooth running of the project was the loss of equipment (pedometers and activity meters) so contingencies need to be built in to deal with such issues.

\subsection{Inspiring users to think about new technologies}

The technology probes and the innovation workshops were successful in generating usercentred design ideas. Moreover, the field testing feedback and the innovation workshops provided consistent messages about the design needs. (Changing the order of the innovation workshop activities had no impact on the ideas generation).

Success with opportunistic exercise technologies will be dependent upon the extent to which they are ubiquitous wearable technology (where data is visible when wanted and hidden when not). For preference these devices should be embedded in existing products that are already carried by the users, to reduce the need to remember and care for an additional object. However the nature of such gadgets needs to be able to adapt to the context in which they are being used.

In providing support and motivation for the users the feedback needs to keep their interest: therefore, rewards and messages linked to their performance need have variation built in to maintain a sense of excitement and anticipation. The motivational messages need to be positive in tone; negative feedback linked to too little exercise is seen as demotivating and likely to lead to avoidance (as detected in Lin et al's (2006) study). 


\section{CONCLUSIONS}

This study has been valuable in providing an insight into some of the issues that need to be considered in the teenage context as opposed to the adult context. One aspect of the technology probes that seemed to have little impact on the participants was the use of persuasion (through the provision of rewards and positive messages for activity done). This is in contrast to the studies of adults that were reviewed. However, we note that the teenage participants were all in good general health and were relatively active. Therefore, they did not need to engage actively in a study like ours for specific health benefits; it may be that with teenagers who are already exhibiting health problems because of lack of exercise would react in a different way to the technology probes and identify different needs. This is an area to explore further. The relationships between personal attitude and desire to change, motivation and persuasion is unclear. Therefore, until the mechanisms at play here are better understood it seems that novel technologies designed to engage users in opportunistic exercise may be ineffective since the fundamental question of what, and who, needs to be designed for remains unanswered.

\section{ACKNOWLEDGEMENTS}

We thank Sunderland City Council for funding this project. We thank the participants and in particular the youth leaders, Pat Garrigan and Katrina Spurs, for their valuable support.

\section{REFERENCES}

Ahtinen, A., Huuskonen, P., \& Häkkilä, J. (2010). Let's all get up and walk to the North Pole. Proceedings of the 6th Nordic Conference on Human-Computer Interaction Extending Boundaries - NordiCHI '10, pp. 3-12. ACM Press, New York.

Arteaga, S.M., Kudeki, M., Woodworth, A., \& Kurniawan, S. (2010). Mobile system to motivate teenagers' physical activity. Proceedings of the 9th International Conference on Interaction Design and Children - IDC '10, pp1-10. ACM Press, New York

Consolvo, S., Everitt, K., Smith, I., \& Landay, J.A. (2006). Design requirements for technologies that encourage physical activity. Proceedings of the SIGCHI conference on Human Factors in computing systems - CHI '06, pp. 457-466. ACM Press, New York

Consolvo, S., Klasnja, P., McDonald, D.W., Avrahami, D., Froehlich, J., LeGrand, L., et al. (2008). Flowers or a robot army?: encouraging awareness \& activity with personal, mobile displays. Proceedings of the 10th international conference on Ubiquitous computing - UbiComp '08 pp. 54-63. ACM Press, New York.

Consolvo, S., Klasnja, P., McDonald, D.W., \& Landay, J.A. (2009). Goal-setting considerations for persuasive technologies that encourage physical activity. Proceedings of the 4th International Conference on Persuasive Technology Persuasive '09, 1. ACM Press, New York.

De Meester, F., Lenthe, F. J. van, Spittaels, H., Lien, N., \& De Bourdeaudhuij, I. (2009). Interventions for promoting physical activity among European teenagers: a systematic review. The international journal of behavioral nutrition and physical activity, 6(1), 82.

Hutchinson, H., Mackay, W., Westerlund, B., et al (2003). Technology Probes: Inspiring Design for and with Families. $\mathrm{CHI} 03$ Proceedings of the SIGCHI conference on Human factors in computing systems. ACM Press, New York

Kowalski, K., Crocker, P., \& Faulkner, R. (1997). Validation of the physical activity questionnaire for older children. Pediatric exercise science, 9(2), 174-186.

Lin, J.J., Mamykina, L., Lindtner, S., Delajoux, G., \& Strub H. B. (2006). Fish "n" Steps: Encouraging Physical Activity with an Interactive Computer Game. In P. Dourish \& A. Friday (Eds.), UbiComp 2006: Ubiquitous Computing, Lecture Notes in Computer Science, Vol. 4206, 261-278. Springer, Berlin.

Sarkin, J.A, Johnson, S.S., Prochaska, J O., \& Prochaska, J.M. (2001) Applying the transtheoretical model to regular moderate exercise in an overweight population: validation of a stages of change measure. Preventive medicine, 33(5), 462469.

Sirard, J.R., \& Barr-Anderson, D.J. (2008). Physical activity in adolescents: from associations to interventions. The Journal of adolescent health, 42(4), 327-328.

Tobe, H. (2005). Scale development for measuring junior high and high school students' enjoyment, benefit psychological barrier for exercise by item response theory. International journal of sport and health science, 3, 129-141.

Toscos, T., Faber, A., Connelly, K., \& Upoma, A.M. (2008). Encouraging Physical Activity in Teens: Can technology help reduce barriers to physical activity in adolescent girls? 2nd International Conference on Pervasive Computing Technologies for Healthcare (Pervasive Healthcare). Tampere, Finland.

Whitehead, J. R. (1995). A study of children's physical self-perceptions using an adapted physical self-perception profile questionnaire. Pediatric Exercise Science, 7(2), 132-151. 